\section{Burnout y factores de riesgo psicosocial en el personal de un hospital de larga estancia}

\author{
Staff burnout and psychosocial risk factors in a \\ long-stay hospital in Spain
}

\section{Burnout e fatores de risco psicossocial em funcionários de um hospital de longa permanência}

Maria-Jose Merino-Plaza 1,2

Francisco-Javier Carrera-Hueso 1

Nuria Arribas-Boscá 1

Amparo Martínez-Asensi 1

Pedro Vázquez-Ferreiro 3

Alberto Vargas-Morales 2

Narjis Fikri-Benbrahim 2

doi: 10.1590/0102-311X00189217

\section{Resumen}

Nuestro objetivo fue evaluar la relación entre los riesgos psicosociales y el burnout en un hospital español de media-larga estancia. Se realizó un estudio transversal en 2017, aplicando la versión española del MBI-HSS y el cuestionario F-Psico 3.1 del Instituto Nacional de Seguridad e Higiene en el Trabajo. Variables predictoras: características sociodemográficas, moduladoras y factores de riesgo psicosocial. Variables resultado: prevalencia de burnout y afectación de sus subescalas. La asociación entre variables se cuantificó con odds ratio. El cansancio emocional se asoció positivamente a los riesgos psicosociales vinculados a carga de trabajo, demandas psicológicas, participación/supervisión, desempeño de rol, relaciones/apoyo social y al consumo de ansioliticos; fueron factores protectores los hijos, sentirse valorado por pacientes y compañeros, satisfacción laboral, optimismo y apoyo social. Las asociaciones halladas para la despersonalización fueron similares, pero más débiles. La baja realización personal se asoció positivamente a los riesgos psicosociales vinculados al tiempo trabajado, autonomía, variedad/contenido del trabajo, desempeño de rol y apoyo social; fue la subescala que mostró mayor número de variables sociodemográficas/moduladoras protectoras: estado civil, tener hijos, trabajar de noche, sentirse valorado por pacientes y familiares, ilusión por el trabajo, apoyo social, autoeficacia y optimismo. Según nuestros resultados, existe asociación entre los riesgos psicosociales y el burnout. Los individuos con mayor satisfacción laboral, autoeficacia y optimismo, afrontan mejor el estrés y son menos vulnerables a los riesgos psicosociales y al burnout
Correspondencia

M-J Merino-Plaza Hospital Doctor Moliner.

Carretera de PortaCoeli s/ $n$, Serra/Comunidad Valenciana 46118, España.

merino_mjo@gva.es

1 Hospital Doctor Moliner, Valencia, España.

2 Universidad de Granada, Granada, España.

${ }_{3}$ Hospital Virxen da Xunqueira, La Coruña, España. 


\section{Introducción}

La Organización Mundial de la Salud (OMS) definió en 1984 los riesgos psicosociales como aquellos aspectos relacionados con la organización, el contenido y la realización del trabajo que pueden afectar al bienestar y la salud del trabajador 1. Cuando las exigencias del trabajo no se adaptan a sus necesidades, expectativas o capacidades, se producen situaciones de riesgo, con posibles consecuencias para las personas y para la organización 2,3. La exposición a factores psicosociales adversos en el entorno laboral produce tensión mental, que si se mantiene en el tiempo, incrementa el riesgo de enfermedades cardiovasculares, musculo-esqueléticas, gastrointestinales y psicológicas 4,5, aumentando también el absentismo, la conflictividad laboral y los abandonos voluntarios de la empresa 6,7,8. Estos desajustes entre el profesional y su entorno se han relacionado con el burnout y la insatisfacción laboral 9,10. Cuanto mayor es la brecha entre la persona y su trabajo (falta de cumplimiento de expectativas, falta de apoyo, conflictos de rol...), mayor es la probabilidad de aparición de burnout.

Clínicamente, el burnout es el estado al que llega el trabajador en respuesta a estresores laborales crónicos 11. Según la perspectiva psicosocial es consecuencia de la interacción dinámica entre el individuo y su entorno laboral 12; el burnout, tiene su origen en el trabajo y no en el trabajador. Este aspecto es importante para enfocar su prevención y tratamiento, identificando las fuentes de estrés y actuando sobre ellas y no dirigiendo la atención únicamente hacia el indivíduo 7.

En sanidad, trabajar con pacientes en circunstancias de ansiedad y dolor, hace que los profesionales estén expuestos a riesgos psicosociales que convierten el desempeño en no gratificante y aumentan el estrés percibido 13,14 .

Desde el punto de vista organizacional, las instituciones sanitarias en muchas ocasiones presentan una cultura resistente al cambio, en lo que respecta a las políticas de gestión de recursos humanos 15, pudiendo generarse un entorno laboral "tóxico" en el colectivo profesional, con consecuencias psicosociales que pueden producir síntomas psicosomáticos e insatisfacción laboral. Según Aiken et al. 16, estas organizaciones con políticas de gestión de recursos humanos inadecuadas a las necesidades asistenciales producen un descenso significativo del rendimiento profesional y aumentan el riesgo de aparición de errores en el desempeño de la actividad, incrementando la morbilidad-mortalidad y dando lugar a lo que podrían llamarse "Hospitales estresados". Por el contrario, existen entornos donde los trabajadores están comprometidos e identificados con su trabajo y ante cargas y factores estresantes similares, no se produce burnout, lo que sugiere que las reacciones frente a una determinada situación, no son las mismas para todos los trabajadores, sino que están condicionadas por características propias del individuo y de la organización 5,13. Ya en los años 1980 se realizaron estudios para identificar los factores responsables de la atracción que ejercían los llamados "Hospitales magnéticos" sobre el personal y se comprobó que esos hospitales presentaban características organizacionales que permitían a las personas desarrollarse profesionalmente y que esas formas de gestión estaban relacionadas con la satisfacción laboral 16,17,18,19. También se observó que en esos entornos, las tasas de mortalidad eran inferiores a las de otros hospitales de su mismo nivel, o sea, que la calidad de los cuidados y la seguridad del paciente se relacionaba con la gestión participativa llevada a cabo en este tipo de centros 16,20,21.

Respecto a nuestro hospital, éste atiende mayoritariamente a pacientes crónicos y de larga evolución, con gran dependencia y discapacidad, o en situaciones clínicas de enfermedad avanzada y, debido a la gran vulnerabilidad de sus pacientes, se puede producir mayor implicación emocional y estrés que en otro tipo de instituciones sanitarias 22. Esta realidad, junto al elevado absentismo laboral del último año (22,55\%), y la falta de investigaciones al respecto, realizadas en hospitales de media-larga estancia, motivó el diseño del presente estudio.

Nuestro hospital pertenece al Sistema Nacional de Salud, universal y público, con una organización jerárquica claramente definida. Las direcciones están sometidas a cambios políticos y por tanto, los estilos directivos varían con el tiempo. En el momento del estudio, la organización era jerarquizada, vertical y sin participación activa de los profesionales en la gestión. Se trataba de una organización burocratizada con una dirección de enfermería poco accesible, de modo que los profesionales recibían escaso reconocimiento y soporte interno. Por el contrario, el trabajo en las unidades se organiza en base a equipos multidisciplinares compuestos por trabajadores de distintas categorías profesionales, lo que permite obtener una visión integral del individuo, no sólo de su situación biomédica completa, sino también de su situación social y funcional. En la atención a enfermos crónicos pluripatológicos, 
cobran mayor importancia las necesidades y prioridades individuales, no sólo médicas, sino también emocionales, sociales, materiales, e incluso espirituales. Estos equipos elaboran una valoración biopsicosocial y un plan de actuación individualizado para cada paciente y revisan periódicamente los objetivos terapéuticos definidos. Este modelo, evita la fragmentación del trabajo, la concentración de poder de decisión sobre ciertas categorías profesionales, e incrementa la eficacia de los cuidados, al realizar una atención centrada en el paciente y potenciar su participación en el proceso asistencial 23.

Nuestro objetivo fue evaluar la relación existente entre el perfil de riesgo psicosocial y el burnout en un hospital de media-larga estancia, analizando las asociaciones entre variables e identificando los factores con mayor impacto sobre el burnout.

\section{Métodos}

\section{Diseño}

Estudio transversal realizado en 2017. Se evaluaron conjuntamente el burnout y el perfil de riesgo psicosocial de los profesionales de un hospital público español de media-larga estancia de 187 camas. La participación fue voluntaria y anónima. Antes de la realización del estudio se obtuvo la aprobación de la Dirección y de las Comisiones de Calidad, Bioética y Docencia e Investigación.

\section{Herramientas}

El instrumento utilizado para evaluar el burnout fue la versión española de la segunda edición del Maslach Burnout Inventory (MBI-HSS) 24, dirigido a profesionales sanitarios. Para determinar la afectación de las subescalas se utilizaron los puntos de corte de Gil-Monte \& Peiró 25.

Para evaluar los riesgos psicosociales se utilizó el cuestionario F-Psico 3.1, diseñado por el Instituto Nacional de Seguridad e Higiene en el Trabajo (INSHT, España) 3, validado y adaptado a nuestro medio por la Universidad de Barcelona 26.

El cuestionario aplicado constó de cuatro partes. La primera contenía las 44 preguntas del cuestionario F-Psico 3.1 que evaluaban, mediante una escala Likert de 5 grados, los nueve factores de riesgo psicosocial considerados: tiempo trabajado (TT), autonomía (AU), carga de trabajo (CT), demandas psicológicas (DPs), variedad/contenido del trabajo (V/C), participación/supervisión (P/S), interés por el trabajador/compensación (IT/C), desempeño de rol (DR) y relaciones/apoyo social (R/AS). La segunda parte recogía las características sociodemográficas de los encuestados (edad, sexo, estado civil, hijos, categoría profesional, tipo de contrato, antigüedad laboral, turnicidad, responsabilidad sobre equipos, contacto directo con pacientes, bajas laborales, enfermedades crónicas, fármacos ansiolíticos). La tercera parte recababa información sobre factores desencadenantes y moduladores del burnout descritos en la literatura 11. Los factores desencadenantes evaluados fueron las relaciones interpersonales entre el profesional y los clientes (valoración por familiares y pacientes) y las relaciones interpersonales en el trabajo (valoración por compañeros y jefes), utilizándose para ello una escala dicotómica. Las variables moduladoras evaluadas fueron: ilusión por el trabajo, satisfacción laboral, apoyo social y ciertas variables personales, como autoeficacia, optimismo y tendencias depresivas, valorándose todas ellas mediante una escala discreta de 1 a 10. La cuarta parte del cuestionario incluía las 22 preguntas del test MBI-HSS que evaluaban, mediante una escala Likert de 7 grados, el burnout y el grado de afectación de sus subescalas Cansancio Emocional (CE), Despersonalización (DP) y Baja Realización Personal (RP).

Muestreo y recogida de datos: no se predeterminó el tamaño muestral. Los cuestionarios se enviaron por correo interno a todo el personal (muestreo no probabilístico-consecutivo), junto a una carta que explicaba los objetivos del estudio y solicitaba la colaboración de los profesionales. El cuestionario fue autoadministrado; las respuestas se recogieron en los buzones de sugerencias para asegurar el anonimato.

Para aplicar el instrumento no se requirió el consentimiento explícito de los participantes; al ser la participación voluntaria y anónima, el responder y entregar la encuesta cumplimentada llevaba implícito dicho consentimiento, y así constaba en la información previa aportada a los trabajadores. 
Variables del estudio: las variables resultado fueron el porcentaje de profesionales con alto grado de afectación de las subescalas de burnout (puntuación $\geq$ a los puntos de corte de Gil-Monte \& Peiró) y el porcentaje de profesionales con burnout franco (alto grado de afectación de las 3 subescalas) 25 . Las variables independientes fueron las características sociodemográficas de los trabajadores, su percepción sobre los factores moduladores y su perfil de riesgo psicosocial.

\section{Análisis de datos}

Los cuestionarios se consideraron válidos si se respondían todas las preguntas de los test F-Psico 3.1 y MBI-HSS.

Para describir las variables cuantitativas se utilizaron medidas de posición (media) y de dispersión (desviación estándar) y para las variables categóricas se emplearon medidas de frecuencia (porcentajes)

Para comprobar la distribución normal de las variables se usó el test de Shapiro-Wilk, utilizando modelos paramétricos si se cumplía o sus equivalentes no paramétricos en caso contrario. En el análisis bivariante se utilizó el test de $\chi^{2}$ para relacionar variables cualitativas, el test t de Student para variables cuantitativas y el test de ANOVA de un factor para variables cuantitativas frente a variables cualitativas $\geq 3$ categorías, con la corrección post hoc de Bonferroni o el test de Kruskal-Wallis, según procediera.

Para analizar la influencia de las variables independientes sobre las variables resultado, se dicotomizaron todas ellas. Para la edad y la antigüedad laboral se utilizó su mediana. Para las variables moduladoras evaluadas mediante escala discreta se creó una nueva variable denominada «alta percepción de la variable», alcanzándose dicho nivel cuando la puntuación era $\geq \mathrm{P}_{75}\left(\mathrm{P}_{75}=\right.$ percentil 75 de la muestra) 27 ( $\mathrm{P}_{75}$ fue 9 para todas las variables consideradas, excepto para la depresión, cuyo $\mathrm{P}_{75}=5$ ). Para cada uno de los riesgos psicosociales se creó una nueva variable, denominada "muy alto riesgo psicosocial”, alcanzándose este nivel cuando las respuestas al cuestionario puntuaban en la zona de riesgo muy elevado del perfil valorativo de la subescala 3. Para las subescalas de burnout se codificó una nueva variable denominada "alta afectación", alcanzándose dicho nivel cuando se superaban los puntos de corte definidos por Gil-Monte \& Peiró 25

Para cuantificar la asociación entre variables, se utilizó la odds ratio (OR) y su intervalo del 95\% de confianza (IC95\%). El análisis ajustado se realizó por regresión logística binaria, incluyendo en el modelo las variables potencialmente confundidoras, según el criterio de Maldonado \& Greeland 28.

El análisis estadístico se realizó con el programa IBM SPSS Statistics para Windows, versión 19.0 (https://www.ibm.com/).

\section{Resultados}

La plantilla del hospital en el momento del estudio era de 322 profesionales, pero sólo devolvieron el acuse de recibo 238; los 84 restantes no recibieron la encuesta por distintos motivos (vacaciones, baja laboral, ausencia justificada...), obteniéndose 111 respuestas válidas (índice de participación = 46,6\%).

La Tabla 1 presenta las variables sociodemográficas y moduladoras consideradas. La muestra fue representativa de la población para edad y sexo, presentando mayor índice de participación el personal fijo, facultativos, personal con responsabilidad sobre dirección de equipos y profesionales con contacto directo con pacientes.

Las puntuaciones medias y la estratificación de las variables moduladoras mostró una plantilla con alta percepción de autoeficacia y apoyo social en más del 50\% de los encuestados, que se consideraba optimista en más del 40\% de los casos, pero sólo un tercio de los encuestados presentó alta satisfacción laboral e ilusión por su trabajo, considerándose valorados por los superiores sólo un $72 \%$ de los profesionales.

En la Tabla 2 se presenta los estadísticos descriptivos y el grado de afectación de las subescalas de burnout, el perfil de riesgo psicosocial de los profesionales y la fiabilidad de las subescalas del cuestionario F-Psico 3.1.

Catorce profesionales mostraron alta afectación de las tres subescalas de burnout (prevalencia = 12,6\%). Las puntuaciones medias y el porcentaje de trabajadores con alta afectación, sitúan a nuestros profesionales en un nivel medio de desgaste profesional. 
Tabla 1

Características sociodemográficas y factores moduladores evaluados.

\begin{tabular}{|c|c|c|}
\hline & $\begin{array}{l}\text { Plantilla hospital } \\
\text { Media (DE) }\end{array}$ & $\begin{array}{l}\text { Respondedores de la encuesta } \\
\text { Media (DE) }\end{array}$ \\
\hline \multirow[t]{2}{*}{ Edad (años) } & $47,5(8,7)$ & $46,8(9,4)$ \\
\hline & n (\%) & n (\%) \\
\hline \multicolumn{3}{|l|}{ Sexo } \\
\hline Hombre & $61(18,9)$ & $16(15,0)$ \\
\hline Mujer & $261(81,1)$ & $91(85,0)$ \\
\hline \multicolumn{3}{|l|}{ Tipo de contrato } \\
\hline Fijo & $90(27,9)$ & $40(36,7)$ \\
\hline No fijo & $232(72,1)$ & $66(63,3)$ \\
\hline \multicolumn{3}{|l|}{ Categoría profesional } \\
\hline Facultativos & $25(7,8)$ & $18(16,8)$ \\
\hline Enfermeros & $101(31,4)$ & $34(31,8)$ \\
\hline Auxiliares enfermería & $90(27,9)$ & $32(29,9)$ \\
\hline Celadores & $47(14,6)$ & $1(1,0)$ \\
\hline Administrativos & $20(6,2)$ & $8(7,5)$ \\
\hline Otros & $39(12,1)$ & $14(13,0)$ \\
\hline \multicolumn{3}{|l|}{ Dirección de equipos } \\
\hline No & $276(85,7)$ & $87(78,4)$ \\
\hline Sí & $46(14,3)$ & $24(21,6)$ \\
\hline \multicolumn{3}{|l|}{ Contacto directo con pacientes } \\
\hline No & $119(37,0)$ & $26(23,4)$ \\
\hline Sí & $203(63,0)$ & $85(76,6)$ \\
\hline Estado civil & ND & \\
\hline Vive en pareja & & $68(63,6)$ \\
\hline No vive en pareja & & $39(36,4)$ \\
\hline ¿Tiene hijos? & ND & \\
\hline No & & $29(26,1)$ \\
\hline Sí & & $82(73,9)$ \\
\hline Antigüedad en el puesto de trabajo (años) & ND & \\
\hline$<5$ & & $47(44,8)$ \\
\hline $5-9$ & & $18(17,1)$ \\
\hline $10-14$ & & $15(14,3)$ \\
\hline $15-19$ & & $12(11,4)$ \\
\hline$\geq 20$ & & $13(12,4)$ \\
\hline ¿Trabaja de noches? & ND & \\
\hline No & & $55(49,5)$ \\
\hline Sí & & $56(50,5)$ \\
\hline ¿Bajas laborales en el último año? & ND & \\
\hline No & & $90(81,1)$ \\
\hline Sí & & $21(18,9)$ \\
\hline ¿Padece enfermedades crónicas? & ND & \\
\hline No & & $82(73,9)$ \\
\hline Sí & & $29(26,1)$ \\
\hline ¿Toma fármacos ansiolíticos? & ND & \\
\hline No & & $93(83,8)$ \\
\hline Sí & & $18(16,2)$ \\
\hline
\end{tabular}

(continua) 
Tabla 1 (continuación)

Plantilla hospital

n (\%)

¿Se siente valorado por los pacientes?

No

Sí

¿Se siente valorado por los familiares?

No

Sí

¿Se siente valorado por los compañeros?

No

Sí

¿Se siente valorado por los superiores?

No

Sí

¿Le ilusiona su trabajo? [media $(D E)=7,3(2,4)$, escala de 0 -10]

$\leq 4$

$5-8$

$\geq 9$

Autoeficacia [media $(D E)=8,3(1,75)$, escala de $0-10$ ]

$\leq 4$

$5-8$

$\geq 9$

Satisfacción laboral [media $(D E)=7,75(1,8)$, escala de 0-10]

$\leq 4$

5-8

$\geq 9$

Optimismo [media $(D E)=8,1(1,98)$, escala de 0-10]

$\leq 4$

$5-8$

$\geq 9$

Grado de depresión [media $(D E)=3,3(2,69)$, escala de 0-10]

$\leq 4$

$5-8$

$\geq 9$

Apoyo social [media $(D E)=8,4(2,14)$, escala de 0-10]

$\leq 4$

$5-8$

$\geq 9$
ND

Respondedores de la encuesta

n (\%)

$26(23,4)$

$85(76,6)$

ND

$16(14,4)$

$95(85,6)$

ND

$12(10,8)$

$99(89,2)$

ND

$31(27,9)$

$80(72,1)$

ND

$11(9,9)$

$63(56,8)$

$37(33,3)$

ND

$4(3,6)$

$49(44,1)$

$58(52,3)$

ND

$6(5,4)$

$64(57,7)$

$41(36,9)$

ND

$6(5,4)$

$55(49,6)$

$50(45,0)$

ND

$78(70,3)$

$28(25,2)$

$5(4,5)$

ND

$69(62,2)$

DE: desviación estándar; ND: no disponible.

En cuanto al perfil de riesgo psicosocial, los factores de mayor riesgo fueron la participación/ supervisión, la carga de trabajo y el desempeño de rol. Respecto a la fiabilidad, las alfa de Cronbach de las subescalas consideradas fueron superiores o muy próximas a 0,7 siendo suficientes para garantizar su fiabilidad. La alfa de Cronbach global del cuestionario F-Psico 3.1 fue de 0,817.

La Tabla 3 presenta el análisis ajustado y la asociación de las variables sociodemográficas/moduladoras con los factores de riesgo psicosocial. Sentirse valorado por los superiores fue factor protector frente a los riesgos relacionados con el interés por el trabajador/compensación y el desempeño de rol. La satisfacción laboral también protegía frente al riesgo relacionado con la participación/supervisión, pero se asoció positivamente al riesgo vinculado al interés por el trabajador/compensación, igual que 
Tabla 2

Estadísticos descriptivos y grado de afectación de las subescalas respecto al burnout y al perfil de riesgo psicosocial de los profesionales.

\begin{tabular}{|c|c|c|}
\hline \multicolumn{3}{|c|}{ Burnout (MBI-HSS) } \\
\hline Puntos de corte & $\begin{array}{l}\text { Grado de afectación de las } \\
\text { subescalas }\end{array}$ & $\begin{array}{l}\text { Frecuencia } \\
\text { n (\%) }\end{array}$ \\
\hline \multicolumn{3}{|c|}{ Cansancio emocional (CE) [media $(D E)=19,82(10,5)$, escala de 0-54 } \\
\hline$<16$ & Bajo & $38(34,2)$ \\
\hline $16-24$ & Medio & $38(34,2)$ \\
\hline$\geq 25$ & Alto & $35(31,5)$ \\
\hline \multicolumn{3}{|c|}{ Despersonalización (DP) [media (DE) = 5,8 $(5,16)$, escala de 0-30] } \\
\hline$<4$ & Bajo & $49(44,1)$ \\
\hline $4-8$ & Medio & $31(27,9)$ \\
\hline$\geq 9$ & Alto & $31(27,9)$ \\
\hline \multicolumn{3}{|c|}{ Realización personal (RP) [media (DE) = 37,86 (6,8), escala de 0-48] } \\
\hline$\geq 40$ & Bajo & $54(48,6)$ \\
\hline $36-39$ & Medio & $19(17,1)$ \\
\hline$<36$ & Alto & $38(34,2)$ \\
\hline Prevalência de burnout n (\%) & $14(12,6)$ & \\
\hline \multicolumn{3}{|c|}{ Factores de riesgo psicosocial evaluados (F-Psico 3.1) } \\
\hline Puntos de corte (INSHT) & Situación del riesgo evaluado & $\begin{array}{l}\text { Frecuencia } \\
\text { n (\%) }\end{array}$ \\
\hline \multicolumn{3}{|c|}{ Tiempo trabajado (IT) [media $(\mathrm{DE})=14,19(10,23)$, escala de $0-37$, a de } \\
\hline \multicolumn{3}{|c|}{ Cronbach $=0,714]$} \\
\hline Percentil $<\mathrm{P}_{65}$ & Situación adecuada & $73(65,8)$ \\
\hline $\mathrm{P}_{65} \leq$ Percentil $<\mathrm{P}_{75}$ & Riesgo moderado & $18(16,2)$ \\
\hline $\mathrm{P}_{75} \leq$ Percentil $<\mathrm{P}_{85}$ & Riesgo elevado & $8(7,2)$ \\
\hline Percentil $\geq \mathrm{P}_{85}$ & Riesgo muy elevado & $12(10,8)$ \\
\hline \multicolumn{3}{|c|}{ Autonomía (AU) [media (DE) = 49,41 $(26,7)$, escala de $0-113$, a de } \\
\hline \multicolumn{3}{|c|}{ Cronbach $=0,863$} \\
\hline Percentil $<\mathrm{P}_{65}$ & Situación adecuada & $84(75,7)$ \\
\hline $\mathrm{P}_{65} \leq$ Percentil $<\mathrm{P}_{75}$ & Riesgo moderado & $10(9,0)$ \\
\hline $\mathrm{P}_{75} \leq$ Percentil $<\mathrm{P}_{85}$ & Riesgo elevado & $5(4,5)$ \\
\hline Percentil $\geq \mathrm{P}_{85}$ & Riesgo muy elevado & $12(10,8)$ \\
\hline \multicolumn{3}{|c|}{ Carga de trabajo $(C T)[$ media $(D E)=47,48(19,04)$, escala de $0-106$, a de } \\
\hline \multicolumn{3}{|c|}{ Cronbach $=0,717]$} \\
\hline Percentil $<\mathrm{P}_{65}$ & Situación adecuada & $52(46,9)$ \\
\hline $\mathrm{P}_{65} \leq$ Percentil $<\mathrm{P}_{75}$ & Riesgo moderado & $14(12,6)$ \\
\hline $\mathrm{P}_{75} \leq$ Percentil $<\mathrm{P}_{85}$ & Riesgo elevado & $15(13,5)$ \\
\hline Percentil $\geq \mathrm{P}_{85}$ & Riesgo muy elevado & $30(27,0)$ \\
\hline \multicolumn{3}{|c|}{$\begin{array}{l}\text { Demandas psicológicas (DPs) [media }(D E)=58,6(18,58) \text {, escala de } 0-112 \text {, } \\
\text { a de Cronbach =0,819] }\end{array}$} \\
\hline Percentil $<\mathrm{P}_{65}$ & Situación adecuada & $60(54,1)$ \\
\hline $\mathrm{P}_{65} \leq$ Percentil $<\mathrm{P}_{75}$ & Riesgo moderado & $15(13,5)$ \\
\hline $\mathrm{P}_{75} \leq$ Percentil $<\mathrm{P}_{85}$ & Riesgo elevado & $17(15,3)$ \\
\hline Percentil $\geq \mathrm{P}_{85}$ & Riesgo muy elevado & $19(17,1)$ \\
\hline \multicolumn{3}{|c|}{ Variedad y contenido del trabajo (VC) [media $(\mathrm{DE})=21,86(11,9)$, escala de } \\
\hline \multicolumn{3}{|c|}{$0-69$, a de Cronbach $=0,689]$} \\
\hline Percentil $<\mathrm{P}_{65}$ & Situación adecuada & $89(80,2)$ \\
\hline $\mathrm{P}_{65} \leq$ Percentil $<\mathrm{P}_{75}$ & Riesgo moderado & $9(8,1)$ \\
\hline $\mathrm{P}_{75} \leq$ Percentil $<\mathrm{P}_{85}$ & Riesgo elevado & $8(7,2)$ \\
\hline Percentil $\geq \mathrm{P}_{85}$ & Riesgo muy elevado & $5(4,5)$ \\
\hline
\end{tabular}

(continua) 
Tabla 2 (continuación)

Factores de riesgo psicosocial evaluados (F-Psico 3.1)

Puntos de corte (INSHT)

Frecuencia

n (\%)
Participación y supervisión (PS) [media (DE) = 40,53 (15,45), escala de 0-87,

a de Cronbach $=0,791]$

Percentil $<\mathrm{P}_{65}$

$\mathrm{P}_{65} \leq$ Percentil $<\mathrm{P}_{75}$

$\mathrm{P}_{75} \leq$ Percentil $<\mathrm{P}_{85}$

Percentil $\geq \mathrm{P}_{85}$

Interés por el trabajador/compensación (ITC) [mdeia $(\mathrm{DE})=39,82(18,13)$,

escala de 0-73, a de Cronbach $=0,708]$

Percentil $<\mathrm{P}_{65}$

$\mathrm{P}_{65} \leq$ Percentil $<\mathrm{P}_{75}$

$\mathrm{P}_{75} \leq$ Percentil $<\mathrm{P}_{85}$

Percentil $\geq \mathrm{P}_{85}$

Desempeño de rol (DR) [media $(D E)=38,36(19,57)$, escala de 0-109, a de

Cronbach $=0,678]$

Percentil $<\mathrm{P}_{65}$

$\mathrm{P}_{65} \leq$ Percentil $<\mathrm{P}_{75}$

$\mathrm{P}_{75} \leq$ Percentil $<\mathrm{P}_{85}$

Percentil $\geq \mathrm{P}_{85}$

Relaciones de apoyo social (RAS) [media $(D E)=23,16(15,08)$, escala de $0-97$, a

de Cronbach $=0,693$

Percentil $<\mathrm{P}_{65}$

$\mathrm{P}_{65} \leq$ Percentil $<\mathrm{P}_{75}$

$\mathrm{P}_{75} \leq$ Percentil $<\mathrm{P}_{85}$

Percentil $\geq P_{85}$

$\begin{array}{cc}\text { Situación adecuada } & 20(18,0) \\ \text { Riesgo moderado } & 9(8,1) \\ \text { Riesgo elevado } & 23(20,7) \\ \text { Riesgo muy elevado } & 59(53,2)\end{array}$

Situación adecuada

$75(67,6)$

$9(8,1)$

$17(15,3)$

$10(9,0)$
$55(49,6)$

$14(12,6)$

$21(18,9)$

$21(18,9)$

DE: desviación estándar; INSHT: Instituto Nacional de Seguridad e Higiene en el Trabajo.

$\begin{array}{cc}\text { Situación adecuada } & 66(59,5) \\ \text { Riesgo moderado } & 9(8,1) \\ \text { Riesgo elevado } & 19(17,1) \\ \text { Riesgo muy elevado } & 17(15,3)\end{array}$

sucedía con las bajas laborales. La ilusión por el trabajo se asoció positivamente al riesgo vinculado a las demandas psicológicas, mientras que una antigüedad en el puesto $<10$ años se asociaba positivamente al riesgo relacionado con la participación/supervisión.

La Tabla 4 presenta el análisis ajustado y la asociación entre las variables sociodemográficas/moduladoras y las subescalas de burnout. Los factores protectores frente al CE fueron tener hijos, sentirse valorado por pacientes y compañeros, satisfacción laboral, optimismo y apoyo social, mientras que tomar ansiolíticos incrementaba la afectación de la subescala. Respecto a la DP, tener hijos, sentirse valorado por los compañeros, disfrutar con el trabajo, autoeficacia, satisfacción laboral y optimismo fueron factores protectores, mientras que trabajar de noches y tomar ansiolíticos se asoció positivamente a la DP. Fueron factores protectores para la RP vivir en pareja, tener hijos, trabajar de noches, sentirse valorado por pacientes y familiares, disfrutar con el trabajo, autoeficacia, optimismo y apoyo social. Respecto al burnout, tener hijos, sentirse valorado por pacientes y familiares, optimismo y apoyo social fueron factores protectores, mientras que tomar ansiolíticos se asoció positivamente al síndrome.

Para analizar la relación existente entre los factores de riesgo psicosocial y el burnout, se realizó el test de ANOVA o Kruskal-Wallis, según procediera, confrontado como variables dependientes las dimensiones del burnout (CE, DP y RP) con cada uno de los factores psicosociales considerados, en función de su nivel de riesgo, como variables independientes.

El CE se asociaba a la carga de trabajo, demandas psicológicas, variedad/contenido del trabajo, participación/supervisión y desempeño de rol.

La DP se asociaba al tiempo trabajado, carga de trabajo, variedad/contenido, desempeño de rol y relaciones y apoyo social.

La RP se asoció al tiempo trabajado, autonomía, variedad/contenido, interés por el trabajador y a las relaciones y apoyo social. 
Asociación de las variables sociodemográficas y moduladoras con los factores de riesgo psicosocial.

\begin{tabular}{|c|c|c|c|c|c|c|c|c|}
\hline & \multicolumn{2}{|c|}{$\begin{array}{c}\text { Demandas } \\
\text { psicológicas (DPs) }\end{array}$} & \multicolumn{2}{|c|}{ Participación/Supervisión (PS) } & \multicolumn{2}{|c|}{$\begin{array}{l}\text { Interés por el trabajador/ } \\
\text { compensación (ITC) }\end{array}$} & \multicolumn{2}{|c|}{$\begin{array}{l}\text { Desempeño } \\
\text { de rol (DR) }\end{array}$} \\
\hline & $\begin{array}{c}\text { Muy alto } \\
\text { riesgo de } \\
\text { afectación/ } \\
\text { Total } \\
\text { n (\%) }\end{array}$ & $\begin{array}{l}\text { OR ajustada } \\
\text { (IC95\%) }\end{array}$ & $\begin{array}{l}\text { Muy alto } \\
\text { riesgo de } \\
\text { afectación/ } \\
\text { Total } \\
\text { n (\%) }\end{array}$ & $\begin{array}{l}\text { OR ajustada } \\
\qquad(\mathrm{IC} 95 \%)\end{array}$ & $\begin{array}{c}\text { Muy alto } \\
\text { riesgo de } \\
\text { afectación/ } \\
\text { Total } \\
\text { n (\%) }\end{array}$ & $\begin{array}{l}\text { OR ajustada } \\
\text { (IC95\%) }\end{array}$ & $\begin{array}{c}\text { Muy alto } \\
\text { riesgo de } \\
\text { afectación/ } \\
\text { Total } \\
\text { n (\%) }\end{array}$ & $\begin{array}{l}\text { OR ajustada } \\
\text { (IC95\%) }\end{array}$ \\
\hline \multicolumn{9}{|l|}{$\begin{array}{l}\text { Antigüedad en } \\
\text { el puesto (años) }\end{array}$} \\
\hline$<10$ & $11 / 64(17,2)$ & 1,8 * & $35 / 64(54,7)$ & $5,1 * *$ & $7 / 64(10,9)$ & 1,1 * & $12 / 64(18,8)$ & 0,8 * \\
\hline$\geq 10$ & $6 / 40(15,0)$ & $(0,2-13,3)$ & $20 / 40(40,0)$ & $(1,1-23,6)$ & $3 / 40(7,5)$ & $(0,1-8,3)$ & $8 / 40(20,0)$ & $(0,2-4,4)$ \\
\hline \multicolumn{9}{|l|}{ Bajas laborales } \\
\hline Sí & $4 / 21(19,0)$ & $1,01 \star \star \star$ & $14 / 21(66,7)$ & $2,8 * \star \star$ & $4 / 21(19,0) \#$ & $8,2 * * *$ & $4 / 21(19,0)$ & $0,7 * \star \star$ \\
\hline No & $15 / 90(16,7)$ & $(0,1-11,3)$ & $45 / 90(50,0)$ & $(0,4-19,2)$ & $6 / 90(6,7)$ & $(1,1-59,1)$ & $17 / 90(18,9)$ & $(0,07-7,4)$ \\
\hline \multicolumn{9}{|l|}{ Valorado por } \\
\hline $\begin{array}{l}\text { Sí } \\
\text { No }\end{array}$ & $\begin{array}{l}14 / 80(16,5) \\
5 / 31(16,1)\end{array}$ & $\begin{array}{c}3,4 \# \# \\
(0,3-37,4)\end{array}$ & $\begin{array}{c}36 / 80(45,0) \# \# \# \\
23 / 31(74,2)\end{array}$ & $\begin{array}{c}0,7 \# \# \\
(0,2-3,1)\end{array}$ & $\begin{array}{l}5 / 80(6,3) \\
5 / 31(16,1)\end{array}$ & $\begin{array}{c}0,15 \# \# \\
(0,03-0,9)\end{array}$ & $\begin{array}{c}10 / 80(12,5) \# \# \# \\
11 / 31(35,5)\end{array}$ & $\begin{array}{c}\mathbf{0 , 1 5} \# \# \\
(0,03-0,7)\end{array}$ \\
\hline \multicolumn{9}{|l|}{$\begin{array}{l}\text { ¿Le ilusiona su } \\
\text { trabajo? }\end{array}$} \\
\hline $\begin{array}{l}\text { Alta } \\
\text { percepción }\end{array}$ & $6 / 37(16,2)$ & $\begin{array}{c}\mathbf{8 , 6 \S} \\
(1,1-65,9)\end{array}$ & $19 / 37(51,4)$ & $\begin{array}{c}0,6 \S \\
(0,2-2,2)\end{array}$ & $13 / 37(13,5)$ & $\begin{array}{c}1,7 \S \\
(0,3-9,7)\end{array}$ & $4 / 37(10,8)$ & $\begin{array}{c}0,8 \S \\
(0,2-4,1)\end{array}$ \\
\hline Resto & $13 / 74(17,7)$ & & $40 / 74(54,1)$ & & $5 / 74(6,9)$ & & $17 / 74(23,0)$ & \\
\hline \multicolumn{9}{|l|}{$\begin{array}{l}\text { Satisfacción } \\
\text { laboral }\end{array}$} \\
\hline $\begin{array}{l}\text { Alta } \\
\text { percepción }\end{array}$ & $5 / 41(12,2)$ & $\begin{array}{c}1,1 \S \\
(0,19-5,8)\end{array}$ & $16 / 41(39,0) * \star$ & $\begin{array}{c}\mathbf{0 , 2} \S \\
(0,05-0,9)\end{array}$ & $7 / 41(17,1)$ & $\begin{array}{c}23,3 \S \\
(1,4-391)\end{array}$ & $5 / 41(12,2)$ & $\begin{array}{c}0,6 \S \\
(0,1-3,3)\end{array}$ \\
\hline Resto & $14 / 70(20,0)$ & & $43 / 70(61,4)$ & & $3 / 70(4,3)$ & & $16 / 70(22,9)$ & \\
\hline
\end{tabular}

IC95\%: intervalo de 95\% de confianza; OR: odds ratio.

* Ajustado para edad, sexo, categoría profesional, tipo de contrato;

** Ajustado para edad, sexo, categoría profesional, disfrutar con el trabajo;

*** Ajustado para edad, sexo, antigüedad en el puesto de trabajo, tipo de contrato;

$\# \mathrm{p}<0,05$;

\#\# Ajustado para edad, categoría profesional, antigüedad en el puesto de trabajo, tipo de contrato;

$\# \#$ \# $<0,001$;

§ Ajustado para edad, sexo, antigüedad en el puesto de trabajo, categoría profesional.

Los resultados del análisis ajustado se presentan en la Tabla 5. Según nuestros datos, la carga de trabajo, demandas psicológicas, participación/supervisión, desempeño de rol y relaciones/apoyo social, se asociaron positivamente al CE. La DP se asoció positivamente a los riesgos relacionados con carga de trabajo, demandas psicológicas, desempeño de rol y relaciones/apoyo social. La baja RP se asoció positivamente a los factores relacionados con el tiempo trabajado, autonomía, variedad/ contenido del trabajo, desempeño de rol y relaciones/apoyo social. Finalmente, el burnout se asoció positivamente a los factores relacionados con tiempo trabajado, autonomía, carga de trabajo, varie$\mathrm{dad} /$ contenido del trabajo, desempeño de rol y relaciones/apoyo social. 
Asociación de las variables sociodemográficas y moduladoras con las subescalas de burnout.

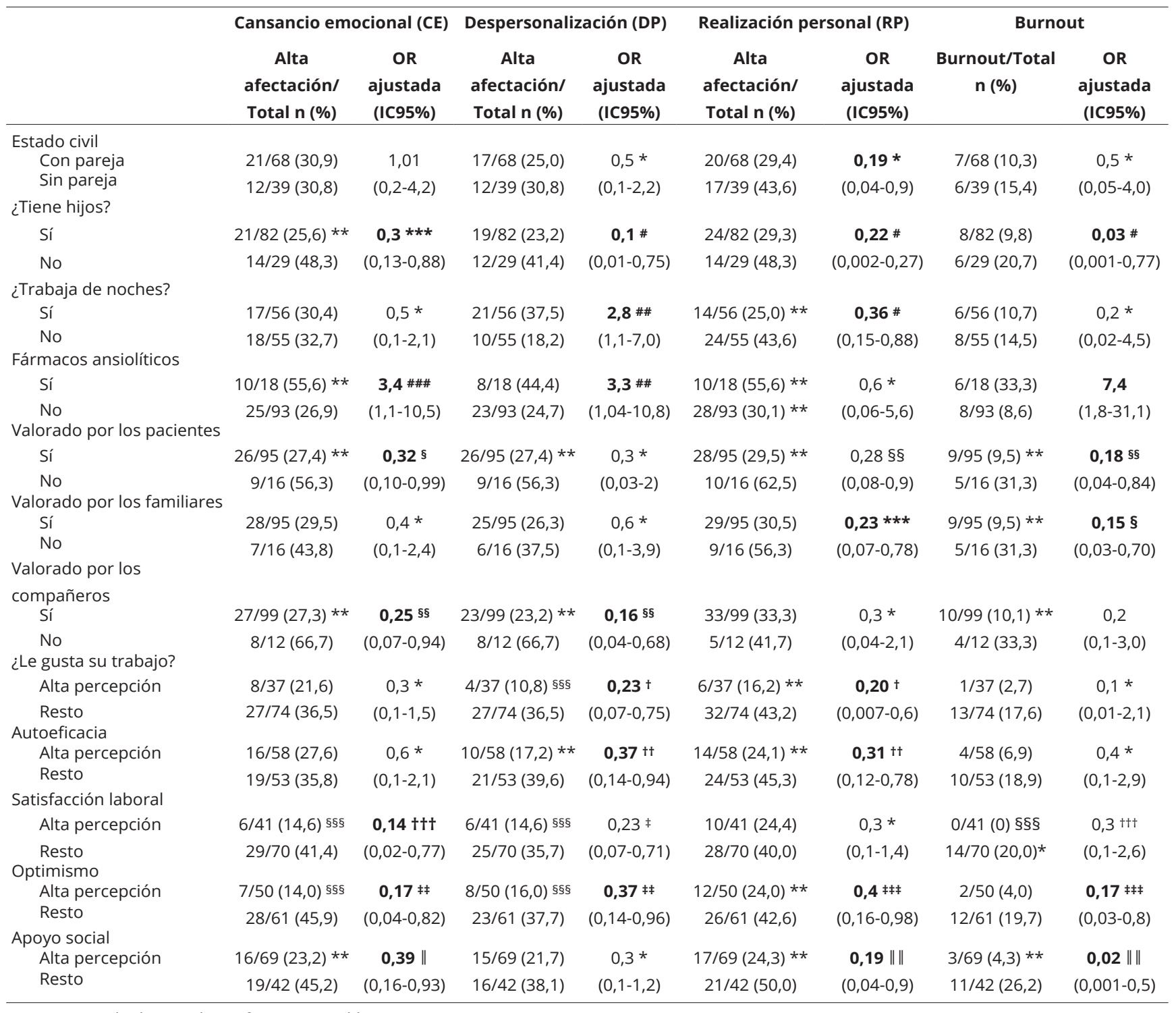

IC95\%: intervalo de 95\% de confianza; OR: odds ratio.

* Ajustado para edad, sexo, antigüedad en el puesto de trabajo, tipo de contrato;

** $\mathrm{p}<0,05$;

*** Ajustado para trabajar de noches, valorado por familiares, valorado por superiores y satisfacción laboral;

\# Ajustado para edad, categoría profesional, antigüedad en el puesto de trabajo, tipo de contrato;

\#\# Ajustado para tener hijos, valorado por pacientes, valorado por superiores y apoyo social;

\#\#\# Ajustado para sexo, bajas laborales, valorado por pacientes y valorado por superiores;

$\S$ Ajustado para tipo de contrato, categoría profesional, valorado por superiores y autoeficacia;

$\S \S$ Ajustado para tener hijos, trabajar de noches, valorado por pacientes y satisfacción laboral;

$\S \S \S<0,001$;

† Ajustado para tipo de contrato, categoría profesional, contacto directo con pacientes y valorado por superiores;

†† Ajustado para tipo de contrato, categoría profesional, valorado por pacientes y valorado por superiores;

††† Ajustado para edad, sexo, antigüedad en el puesto de trabajo, categoría professional;

¥ Ajustado para tipo de contrato, categoría profesional, valorado por familiares y valorado por superiores;

¥¥ Ajustado para edad, sexo, categoría profesional, tipo de contrato;

$\ddagger \ddagger \ddagger$ Ajustado para tener hijos, valorado por pacientes, valorado por superiores y trabajar de noches;

$\|$ Ajustado para tener hijos, trabajar de noches, contacto directo con pacientes y valorado por familiares;

| | | A Ajustado para edad, tipo de contrato, valorado por pacientes y autoeficacia. 


\section{Tabla 5}

Asociación de los factores de riesgo psicosocial con las subescalas de burnout.

\begin{tabular}{|c|c|c|c|c|c|c|c|c|}
\hline \multirow{2}{*}{$\begin{array}{l}\text { Factores de riesgo } \\
\text { psicosocial }\end{array}$} & \multicolumn{2}{|c|}{ Cansancio emocional (CE) } & \multicolumn{2}{|c|}{ Despersonalización (DP) } & \multicolumn{2}{|c|}{ Realización personal (RP) } & \multicolumn{2}{|c|}{ Burnout } \\
\hline & $\begin{array}{c}\text { Alta } \\
\text { afectación/ } \\
\text { Total } \\
\text { n (\%) }\end{array}$ & $\begin{array}{c}\text { OR } \\
\text { ajustada } \\
\text { (IC95\%) }\end{array}$ & $\begin{array}{c}\text { Alta } \\
\text { afectación/ } \\
\text { Total } \\
\mathbf{n}(\%)\end{array}$ & $\begin{array}{c}\text { OR } \\
\text { ajustada } \\
\text { (IC95\%) }\end{array}$ & $\begin{array}{c}\text { Alta } \\
\text { afectación/ } \\
\text { Total } \\
\text { n (\%) }\end{array}$ & $\begin{array}{c}\text { OR } \\
\text { ajustada } \\
\text { (IC95\%) }\end{array}$ & $\begin{array}{c}\text { Burnout/ } \\
\text { Total } \\
\text { n (\%) }\end{array}$ & $\begin{array}{c}\text { OR } \\
\text { ajustada } \\
\text { (IC95\%) }\end{array}$ \\
\hline \multicolumn{9}{|l|}{ Tiempo trabajado (TT) } \\
\hline Muy alto riesgo & $4 / 12(33,3)$ & $\begin{array}{c}1,3 * \\
0,05-11,9)\end{array}$ & $4 / 12(33,3)$ & $\begin{array}{c}2,8 * \\
(0,1-59)\end{array}$ & $8 / 12(66,7)$ ** & $\begin{array}{l}20,0 * * * \\
(3,8-107)\end{array}$ & $3 / 12(25,0)$ ** & $\begin{array}{c}9,4 * * * \\
(1,4-70,1)\end{array}$ \\
\hline Resto & $31 / 99(31,3)$ & & $27 / 99(27,3)$ & & $30 / 99(30,3)$ & & $11 / 99(11,1)$ & \\
\hline \multicolumn{9}{|l|}{ Autonomía (AU) } \\
\hline Muy alto riesgo & $6 / 12(50,0)$ & $\begin{array}{c}3,1 \text { * } \\
(0,4-22,6)\end{array}$ & $5 / 12(41,7)$ & $\begin{array}{c}2,2 * \\
(0,2-20,5)\end{array}$ & $8 / 12(66,7) * *$ & $\begin{array}{c}\mathbf{2 2 , 4} \# \\
(1,1-454)\end{array}$ & $4 / 12(33,3)$ ** & $\begin{array}{c}\mathbf{2 4 , 1} \text { \# } \\
(1,1-568)\end{array}$ \\
\hline $\begin{array}{l}\text { Resto } \\
\text { Carga de trabajo } \\
\text { (CT) }\end{array}$ & $29 / 99(29,3)$ & & $26 / 99(26,3)$ & & $30 / 99(30,3)$ & & $10 / 99(10,1)$ & \\
\hline Muy alto riesgo & $20 / 30(66,7) * *$ & $\begin{array}{c}11,2 \text { * } \\
(1,8-69,8)\end{array}$ & $16 / 30(53,3)$ * & $\begin{array}{c}\mathbf{8 , 1} \text { * } \\
(1,3-52,7)\end{array}$ & $11 / 30(36,7)$ & $\begin{array}{c}0,9 * * * \\
(0,3-2,4)\end{array}$ & $8 / 30(26,7)$ ** & $\begin{array}{c}3,8 * * * \\
(1,1-13,7)\end{array}$ \\
\hline Resto & $15 / 81(18,5)$ & & $15 / 81(18,5)$ & & $27 / 81(33,3)$ & & $6 / 81(7,4)$ & \\
\hline \multicolumn{9}{|c|}{$\begin{array}{l}\text { Demandas psicológicas } \\
\text { (DPs) }\end{array}$} \\
\hline Muy alto riesgo & $10 / 19(52,6) * *$ & $\begin{array}{c}6,6 \# \# \\
(1,1-39,3)\end{array}$ & $8 / 19(42,1)$ & $\begin{array}{c}3,8 \# \# \# \\
(1,2-12,6)\end{array}$ & $6 / 19(31,6)$ & $\begin{array}{l}1,3 * * * \\
(0,4-4,3)\end{array}$ & $4 / 19(21,1)$ & $\begin{array}{c}3,4 * * * \\
(0,7-16,7)\end{array}$ \\
\hline Resto & $25 / 92(27,2)$ & & $23 / 92(25,0)$ & & $32 / 92(34,8)$ & & $10 / 92(10,9)$ & \\
\hline \multicolumn{9}{|c|}{ Variedad ycontenido (VC) } \\
\hline Muy alto riesgo & $5 / 5(100,0) \S$ & $\begin{array}{c}0,3 * * * \\
(0,05-2,3)\end{array}$ & $5 / 5(100,0) \S$ & $\begin{array}{c}3,2 * * * \\
(0,3-34,5)\end{array}$ & $4 / 5(80,0) \S$ & $\begin{array}{c}11,8 \S \S \\
(1,1-141)\end{array}$ & $4 / 5(80,0) \S$ & $\begin{array}{l}32,9 * * * \\
(2,6-412)\end{array}$ \\
\hline \multicolumn{9}{|c|}{$\begin{array}{l}\text { Participación /Supervisión } \\
\text { (PS) }\end{array}$} \\
\hline Muy alto riesgo & $55 / 59(42,4) * \star$ & $\begin{array}{c}\mathbf{4 , 8} 8 \S \S \\
(1,6-14,4)\end{array}$ & $16 / 59(27,1)$ & $\begin{array}{c}1,4 \text { * } \\
(0,3-6,9)\end{array}$ & $20 / 59(33,9)$ & $\begin{array}{l}1,02 \text { *** } \\
(0,4-2,6)\end{array}$ & $7 / 59(11,9)$ & $\begin{array}{l}1,1 * * * \\
(0,3-4,3)\end{array}$ \\
\hline Resto & $10 / 52(19,2)$ & & $15 / 52(28,8)$ & & $18 / 52(34,6)$ & & $7 / 52(13,5)$ & \\
\hline \multicolumn{9}{|c|}{ Desempeño de rol (DR) } \\
\hline Muy alto riesgo & $13 / 21(61,9) * *$ & $\begin{array}{c}\mathbf{6 , 4} \text { * } \\
(1,03-40)\end{array}$ & $12 / 21(57,1)$ ** & $\begin{array}{c}6,2+ \\
(2-20,2)\end{array}$ & $11 / 21(52,4) * *$ & $\begin{array}{c}\mathbf{2 , 9} \\
(1,1-8,3)\end{array}$ & $7 / 21(33,3) \S$ & $\begin{array}{c}\mathbf{6 , 0}+ \\
(1,4-25,2)\end{array}$ \\
\hline \multicolumn{9}{|c|}{$\begin{array}{l}\text { Relaciones /Apoyo social } \\
\text { (RAS) }\end{array}$} \\
\hline Muy alto riesgo & $9 / 17(52,9) * *$ & $\begin{array}{c}15,7^{\dagger \dagger} \\
(1,3-186)\end{array}$ & $8 / 17(47,1) * *$ & $\begin{array}{c}\mathbf{4 , 4} \# \# \# \\
(1,3-15,1)\end{array}$ & $9 / 17(52,9) * *$ & $\begin{array}{c}3,8 \mathrm{ttt} \\
(1,2-12,3)\end{array}$ & $5 / 17(29,4) * *$ & $\begin{array}{c}\mathbf{4 , 2} \# \# \# \\
(1,2-15,6)\end{array}$ \\
\hline Resto & $26 / 94(27,7)$ & & $23 / 94(24,5)$ & & $29 / 94(30,9)$ & & $9 / 94(9,6)$ & \\
\hline
\end{tabular}

IC95\%: intervalo de 95\% de confianza; OR: odds ratio.

* Ajustado para edad, sexo, hijos y valorado por superiores;

$* * p<0,05$

*** Ajustado para categoría profesional, hijos, responsabilidad sobre dirección de equipos y trabaja de noches;

\# Ajustado para edad, categoría profesional, hijos y valorado por compañeros;

\#\# Ajustado para edad, categoría profesional, hijos y valorado por pacientes;

\#\#\# Ajustado para categoria professional, régimen de trabajo, estado civil y años de antigüedad laboral;

$\S \mathrm{p}<0,001$;

$\S \S$ Ajustado para bajas laborales, valorado por superiores, autoeficacia y grado de depresión;

$\S \S \S$ Ajustado para categoría profesional, hijos, trabaja de noches y valorado por pacientes;

† Ajustado para categoría profesional, hijos, valorado por superiores y apoyo social;

${ }^{\dagger \dagger}$ Ajustado para edad, bajas laborales, responsabilidad sobre equipos y grado de depresión;

${ }^{\dagger \dagger \dagger}$ Ajustado para trabaja de noches, bajas laborales, responsabilidade sobre equipos y grados de depresión. 


\section{Discusión}

Nuestros profesionales se mostraron afectados fundamentalmente por estresores relacionados con aspectos organizacionales y de gestión (desequilibrio en la asignación de recursos, escasa participación en la toma de decisiones, alteración de turnos de trabajo para cubrir incidencias, sistemas de turnos rotatorios desequilibrados, sobrecarga de trabajo en ciertas unidades, falta de apoyo y reconocimiento, escasa participación de los profesionales en la definición de objetivos...). Estos “estresores" dominaron sobre los que podemos considerar más específicos de unidad de trabajo o de factores individuales de la persona.

La tasa de participación fue próxima al 50\%, similar a otros estudios realizados mediante cuestionarios autoadministrados y anónimos 4,7, aunque algunos autores, en el contexto de envíos personalizados o revisiones laborales, obtuvieron tasas de respuesta superiores al 65\% 9,10.

No existe un criterio unánime sobre los puntos de corte a utilizar para diagnosticar el burnout y calcular su prevalencia, por lo que el MBI-HSS se ha adaptado a distintos idiomas y culturas para evitar sesgos de interpretación 29. En nuestro estudio se utilizaron los puntos de corte definidos por Gil-Monte \& Peiró 25 para una muestra multiocupacional española. Las puntuaciones obtenidas sitúan a nuestros profesionales en un nivel medio de desgaste profesional, similar al de otros estudios realizados en hospitales de agudos, siendo el CE y la RP las subescalas más afectadas entre nuestros profesionales. Sin embargo, la prevalencia de burnout en el presente estudio fue sensiblemente superior a la objetivada en el estudio transversal realizado en 2014, habiendo pasado de una tasa del 5\% al 12,6\%. Existen estudios que relacionan el grado de burnout de los profesionales con los estilos de dirección y la cultura organizacional 30,31 y que podrían explicar el incremento de la prevalencia de burnout observado, ya que durante el periodo considerado la dirección del hospital cambió de un estilo participativo, a otro más vertical y jerarquizado.

En el presente estudio, vivir en pareja se asoció positivamente a la RP. Tener hijos fue factor protector para todas las subescalas, probablemente porque la implicación del sujeto con la familia le proporciona mayor capacidad para afrontar problemas y conflictos emocionales 11,32. El CE se asoció positivamente al consumo de ansiolíticos, hecho también descrito en otros trabajos 4,15,33, pero al tratarse de un estudio transversal, no puede establecerse relación causal.

Trabajar de noche y consumir ansiolíticos incrementó el riesgo de DP, hallazgo también descrito por otros autores 4,11. Los turnos de trabajo rotatorios y los horarios irregulares originan alteraciones del sueño que producen cansancio, nerviosismo, trastornos digestivos y de los ritmos biológicos y además dificultan las relaciones socio-familiares 15,34,35. Por outra parte, el trabajo a turnos produce un mayor número de problemas laborales (absentismo, menor rendimiento y accidentes de trabajo) 36, lo que incrementa el riesgo de aparición de burnout. Existen estudios que correlacionan el absentismo con los niveles de estrés de la organización 22,37.

Según la bibliografía, son factores protectores frente al burnout: el apoyo social, retroalimentación recibida, autonomía, disponibilidad de recursos y variables personales como autoeficacia, buenas relaciones interpersonales y compromiso con el trabajo. Cañadas observó que los profesionales con personalidad positiva, optimismo y autoeficacia eran menos propensos a "quemarse" 32. Según diversos autores, los principales factores desencadenantes son ambigüedad y conflicto de rol, sobrecarga laboral física y/o mental, falta de equidad, conflictos interpersonales, falta de control del trabajador sobre su actividad y cultura despersonalizadora de la organización 4,11,32,38. Piko halló fuertes relaciones entre insatisfacción laboral y CE 39. Según nuestros resultados, sentirse valorado por pacientes, familiares y compañeros, disfrutar con el trabajo, autoeficacia, satisfacción laboral, optimismo y apoyo social actuaron como factores protectores. Estas variables amortiguan el estrés percibido e influyen sobre las estrategias de afrontamiento, mejorando el estado de ánimo, la motivación y la autoestima 32. La autoeficacia actuó como factor protector frente a la DP y la RP. El optimismo se asoció negativamente a todas las subescalas del burnout, hecho también descrito por Grau 4. Garrosa et al. 40 comprobó que el optimismo y la competencia emocional se asociaban positivamente con el compromiso organizacional y la prevención del desgaste profesional.

Sentirse valorado por los superiores fue factor protector frente al desempeño de rol y el interés por el trabajador/compensación. Este aspecto es importante, ya que en ocasiones, el trabajo de enfermería está poco reconocido, tanto a nivel directivo, como por otros profesionales del equipo multidisciplinar 41 . 
La satisfacción laboral fue un factor protector frente al riesgo relacionado con la participación/ supervisión. Este hallazgo corrobora la relación positiva existente, según Mansilla Izquierdo 35, entre el grado de control del profesional sobre su trabajo y su satisfacción laboral. La participación en la toma de decisiones es un importante factor de motivación e implicación en la actividad laboral y contribuye a la formación y al crecimiento de los profesionales. Por el contrario, la falta de participación origina en el trabajador una falta de control sobre sus propias condiciones laborales, habiendo sido éste, el principal riesgo psicosocial que afectó a nuestros trabajadores.

Según la literatura, los trabajadores con bajas médicas, perciben menor apoyo organizacional 5. En nuestro estudio, las bajas médicas y la satisfacción laboral se asociaron positivamente al factor vinculado al interés por el trabajador/compensación, mientras que la ilusión por el trabajo, se asoció positivamente al riesgo vinculado a las demandas psicológicas. Estos hallazgos apoyan la teoría de que los sujetos sobre-comprometidos se exponen frecuentemente a altas demandas en el trabajo y exageran sus esfuerzos más allá de lo necesario, siendo más vulnerables y presentando mayor riesgo de sufrir tensión laboral por su percepción de un intercambio asimétrico 42. Esta situación, a largo plazo, puede producir estrés, insatisfacción y una percepción adversa de los riesgos psicosociales, incrementando el riesgo de burnout 5 .

Los riesgos a los que están expuestos los trabajadores, si la organización del trabajo no es adecuada, no son tan evidentes como los accidentes laborales o las enfermedades profesionales, pero son igual de reales 42,43 . En nuestro estudio, la excesiva carga de trabajo se asoció al CE, DP y burnout, mientras que el desempeño de rol y las relaciones/apoyo social se asociaron a todas las subescalas. La indefinición del puesto, los conflictos, ambigüedad, sobrecarga de rol y malas relaciones interpersonales, incrementaron el riesgo de burnout. Según la literatura, los conflictos que surgen entre las distintas categorías profesionales por ambigüedad de rol se relacionan con la aparición de estrés 44 . En nuestro estudio, la asociación más fuerte se observó entre relaciones/apoyo social y CE, resultados también descritos por otros autores 43,45 .

Las demandaspsicológicasse asociaronalCEyDP, resultados halladostambiénen otros trabajos 45,46. La presión asistencial por la carga de trabajo y las demandas psicológicas, es un fuerte estresor, pero puede resultar más estresante sentir que el esfuerzo personal no es apreciado, haciendo que la carga laboral se viva mucho más intensamente e incrementando el estrés percibido.

La participación/supervisión se asoció al CE, mientras que tiempo trabajado, autonomía y varie$\mathrm{dad} /$ contenido del trabajo se asociaron a la RP. La falta de participación del trabajador y el exceso de control por parte de los supervisores incrementó el CE, mientras que la falta de conciliación de la vida personal y laboral, la falta de autonomía y las tareas monótonas afectaron principalmente a la $\mathrm{RP}$, resultados similares a los hallados por otros autores 39 . Esto sugiere que las tareas monótonas son poco motivadoras y que la falta de autonomía y el autoritarismo son una fuente de estrés.

Nuestros resultados indican que los riesgos psicosociales no sólo disminuyen la calidad de vida del trabajador, sino que si no se gestionan bien, generan burnout 47 . El entorno sanitario, presenta características cambiantes que hacen que el profesional deba adaptarse continuamente e incrementan el estrés laboral. Existen diferentes variables que lo condicionan como son: nuevas demandas asistenciales, indefinición y ambigüedad del rol, crisis del sistema, altos niveles de absentismo laboral, baja implicación, falta de motivación, poca conciencia de los gestores sobre el problema y en muchas ocasiones, falta de soluciones desde la organización. Por otra parte, entre los profesionales sanitarios que trabajan en instituciones gestionadas con modelos jerarquizados es relativamente frecuente la existencia de una baja implicación en la consecución de los objetivos establecidos por la institución 48 , debido entre otras causas, a un escaso nivel de autonomía por la dependencia estructural y jerárquica, escaso reconocimiento, indefinición de funciones, ausencia de mecanismos de promoción interna, incomodidad en el trabajo, turnos lesivos, elevada presión asistencial y en muchas ocasiones, ausencia de apoyo de la dirección 49. Estas situaciones crean indefinición y actitudes de baja cohesión en los equipos de trabajo, que pueden deteriorar la percepción de la eficacia colectiva, afectar al rendimiento del grupo e influir en la percepción del apoyo social y en el clima laboral. Según algunos autores, existe un proceso de contagio del burnout en los equipos de trabajo. Si el ambiente es tóxico, aparecen alteraciones en la dinámica laboral que generan estrés. El contagio de los síntomas del burnout estaría vinculado al contagio de emociones, actitudes y al aprendizaje de las conductas de los compañeros 50 , de modo que los profesionales que perciben altos niveles de burnout en su entorno, son más 
propensos a sufrirlo. Por eso, la reducción de la presión laboral, junto con un incremento en la autonomía y la equidad, unido a una política más incentivadora, pueden ser útiles para prevenir el síndrome. El reconocimiento también juega un papel importante, ya que hace que los profesionales reciban feedback de la labor realizada y se sientan valorados y respetados.

El estrés crónico disminuye las defensas psicológicas e inmunológicas y hace más vulnerable al trabajador. Aproximadamente el 8\% de las enfermedades ocupacionales del sector sanitario están relacionadas con el burnout. Para la OMS, los profesionales son un recurso fundamental; la eficacia del sistema depende de sus conocimientos, destrezas y motivación. Ya en 1994, consideró el burnout un serio problema 51 y en su informe sobre la mejora del rendimiento de los sistemas sanitarios 52 invocaba al burnout como responsable de la crisis motivacional del personal sanitario. En el sector público, la desmotivación se relaciona con la falta de mecanismos de promoción interna, burocratización y falta de participación de los profesionales en los mecanismos de gestión. La carga de trabajo y el estrés de rol incrementan el estrés y reducen la satisfacción, influyendo a su vez sobre las relaciones interpersonales. Estos aspectos disminuyen el rendimiento laboral y desdibujan el contexto que mantiene al profesional en el ejercicio vivo y activo de su profesión. Pero la realidad psicosocial, no sólo depende de las condiciones laborales, sino de cómo las percibe el trabajador 5,13. Ante una misma realidad, hay profesionales que se ven afectados, mientras que otros, la perciben como un reto que les permite demostrar sus capacidades, auto-motivándose e incrementando su satisfacción laboral 14. Cuando las demandas exceden las capacidades adaptativas individuales, se producen alteraciones psicológicas y físicas que aumentan el riesgo de enfermar, mientras que si la persona tiene éxito en su esfuerzo adaptativo, mejoran sus parámetros biológicos y aumenta su bienestar subjetivo 53. Para algunos individuos, un trabajo activo, con alta demanda y alto control, promueve nuevas habilidades y conductas de afrontamiento, aspecto a tener en cuenta para asignar a los profesionales un puesto de trabajo acorde a sus capacidades.

Evidenciar empíricamente las relaciones existentes entre el burnout, los factores de riesgo psicosocial y las variables moduladoras, permite justificar y orientar las intervenciones para prevenir el síndrome. Por eso, es fundamental conocer las relaciones existentes entre el burnout y sus variables asociadas, para comprender mejor el síndrome e intervenir de manera eficaz, siendo importantes las actuaciones sobre la cultura organizacional y estilos de liderazgo. El desarrollo organizacional es un proceso largo, orientado a mejorar los procesos sociales $54,55 \mathrm{y}$, según algunos autores, influye en la $\mathrm{RP}$ y reduce el riesgo de burnout 30 .

Entre las limitaciones de nuestro estudio, cabe destacar su diseño transversal, que no permite extraer conclusiones de causalidad ni direccionalidad de las relaciones entre las variables estudiadas. Sería necesario realizar estudios longitudinales para analizar dichas relaciones. Existe un posible sesgo de selección, ya que al ser la participación voluntaria y anónima, los profesionales más proactivos suelen ser también los más participativos. No ha sido posible realizar un análisis más profundo de las características organizacionales del hospital donde se desarrolló el estudio. Finalmente, el bajo número de respuestas válidas, dado el pequeño tamaño de la plantilla, no permite extrapolar nuestros resultados.

Como conclusión, según nuestros resultados, existe asociación entre los factores de riesgo psicosocial y el burnout. Los individuos con mayor satisfacción laboral, autoeficacia y optimismo, afrontan mejor el estrés y son menos vulnerables a los riesgos psicosociales y al burnout. 


\section{Colaboradores}

M.-J. Merino-Plaza ha contribuido en la concepción y diseño del trabajo, análisis e interpretación de los datos, escritura y revisión del artículo; responsable y garante de que todos los aspectos del manuscrito han sido revisados y discutidos entre los autores. F.-J Carrera-Hueso ha colaborado en el diseño del trabajo, análisis de los datos, escritura y revisión del artículo y aprobación de la versión final para su publicación. N. Arribas-Boscá ha colaborado en el diseño del trabajo, interpretación de los datos, escritura y revisión del artículo y aprobación de la versión final para su publicación. A. MartínezAsensi, P. Vázquez-Ferreiro y A. Vargas-Morales han colaborado en la realización del estudio, interpretación de los resultados, escritura y revisión del artículo y aprobación de la versión final para su publicación. N. Fikri-Benbrahim supervisó el estudio, interpretación de los resultados, escritura y revisión del artículo y aprobación de la versión final para su publicación.

\section{Agradecimientos}

A los miembros de la Comisión de Calidad, por los años de colaboración y esfuerzo para mejorar día a día la calidad asistencial. Este artículo forma parte de la tesis doctoral de M. J. Merino-Plaza Calidad Asistencial y Seguridad del Paciente en un Hospital de Media-larga Estancia, Programa de Farmacia Social, Universidad de Granada, España.

\section{Referencias}

1. Organización Internacional del Trabajo; Organización Mundial de la Salud. Factores psicosociales en el trabajo: naturaleza, incidencia y prevención. Informe del Comité mixto OIT/ OMS sobre medicina del trabajo. Geneva: Organización Internacional del Trabajo/Organización Mundial de la Salud; 1988.

2. Leka S, Cox T, Jain A, Hassard J, Ertel M, Stilijanow U, et al. Guidance on the European Framework for Psychosocial Risk Management: a resource for employers and worker representatives. Geneva: World Health Organization; 2008.

3. Instituto Nacional de Seguridad e Higiene en el Trabajo. F-PSICO. Factores psicosociales. Método de evaluación. Versión 3.1. http:// www.insht.es/portal/site/Insht/menuite m.1f1a3bc79ab34c578c2e8884060961ca/?vgn extoid $=88 \mathrm{e} 2 \mathrm{ce} 8 \mathrm{~d} 3 \mathrm{ca} 16310 \mathrm{VgnVCM} 10000081$ 30110aRCRD\&vgnextchannel=ac18b12ff8d8 1110VgnVCM100000dc0ca8c0RCRD (accedido el 27/Feb/2018).

4. Moreno-Jiménez B, Baez-León C. Factores y riesgos psicosociales, formas, consecuencias, medidas y buenas prácticas. Madrid: Instituto Nacional de Seguridad e Higiene en el Trabajo; 2010.

5. Chirico F. Job stress models for predicting burnout syndrome: a review. Ann Ist Super Sanita 2016; 52:443-56.

6. Vignoli M, Guglielmi D, Bonfiglioli R, Violante FS. How job demands affect absenteeism? The mediating role of work-family conflict and exhaustion. Int Arch Occup Environ Health 2016; 89:23-31.

7. Grau A, Suñer R, García MM. Desgaste profesional en el personal sanitario y su relación con los factores personales y ambientales. Gac Sanit 2005; 19:463-70.

8. García JM, Moreno LL, Díaz MJ, Valdehita SR. Relación entre factores psicosociales adversos, evaluados a través del cuestionario multidimensional Decore, y salud laboral deficiente. Psicothema 2007; 19:95-101.

9. Escribà-Agüir V, Artazcoz L, Pérez-Hoyos S. Efecto del ambiente psicosocial y de la satisfacción laboral en el síndrome de burnout en médicos especialistas. Gac Sanit 2008; 22:300-8.

10. Hocine MN, Aït Bouziad K, Légeron P, Dab W, Saporta G. How to identify and prioritize psychosocial factors impacting stress level. PLoS One 2016; 11:e0157078.

11. Maslach C, Schaufeli WB, Leiter MP. Job burnout. Annu Rev Psychol 2001; 52:397-422.

12. Maslach C, Leiter MP. Early predictors of job burnout and engagement. J Appl Psychol 2008; 93:498-12.

13. Gil-Monte PR. Algunas razones para considerar los riesgos psicosociales en el trabajo y sus consecuencias en la salud pública. Rev Esp Salud Pública 2009; 83:169-73. 
14. Privitera MR, Rosenstein AH, Plessow F, LoCastro TM. Physician burnout and occupational stress: an inconvenient truth with unintended consequences. J Hosp Adm 2015; 4:27-35.

15. Mingote Adán JC, Moreno Jiménez B, Herrer M. Desgaste profesional y salud de los profesionales médicos: revisión y propuestas de prevención. Med Clin (Barc) 2004; 123:26570.

16. Aiken LH, Clarke SP, Sloane DM. Hospital staffing, organization, and quality of care: cross-national findings. Int J Qual Health Care 2002; 14:5-14

17. Magnet hospitals. Attraction and retention of professional nurses. Task Force on Nursing Practice in Hospitals. American Academy of Nursing. ANA Publ 1983; (G-160):i-xiv, 1-135.

18. Havens DS, Aiken LH. Shaping systems to promote desired outcomes. The magnet hospital model. J Nurs Adm 1999; 29:14-20.

19. Aiken LH, Patrician PA. Measuring organizational traits of hospitals: the Revised Nursing Work Index. Nurs Res 2000; 49:146-53.

20. Aiken LH, Smith HL, Lake ET. Lower Medicare mortality among a set of hospitals known for good nursing care. Med Care 1994; 32:77187.

21. Aiken LH, Sermeus W, den Heede KV, Sloane DM, Busse R, McKee M, et al. Patient safety, satisfaction, and quality of hospital care: cross sectional surveys of nurses and patients in 12 countries in Europe and the United States. BMJ 2012; 344:e1717.

22. Acinas MP. Burn-out y desgaste por empatía en profesionales de cuidados paliativos. Revista Digital de Medicina Psicosomática y Psicoterapia 2012; 2:1-22.

23. López Almazán C. Atención a personas con enfermedades crónicas una estrategia para la gestión por procesos en un hospital de media y larga estancia.Valencia: Escuela Valenciana de Estudios de la Salud; 2008.

24. Maslach C, Jackson SE. Maslach burnout inventory. 2nd Ed. Palo Alto: Consulting Psychologists Press; 1986.

25. Gil-Monte PR, Peiró JM. Un estudio comparativo sobre criterios normativos y diferenciales para el diagnóstico del síndrome de quemarse por el trabajo (burnout) según el MBI-HSS en España. Revista de Psicología del Trabajo y de las Organizaciones 2000; 16:135-49.

26. Ferrer R, Guilea G, Peró M. Propiedades psicométricas del instrumento de valoración de riesgos psicosociales del Instituto Nacional de Seguridad e Higiene en el Trabajo (FPSICO). Barcelona: Instituto Nacional de Seguridad e Higiene en el Trabajo; 2011

27. Robles-García M, Dierssen-Sotos T, Martínez-Ochoa E, Herrera-Carral P, Díaz-Mendi AR, Llorca-Díaz J. Variables relacionadas con la satisfacción laboral: un estudio transversal a partir del modelo EFQM. Gac Sanit 2005; 19:127-34.

28. Maldonado G, Greenland S. Simulation study of confounder-selection strategies. Am J Epidemiol 1993; 138:923-36.
29. Loera B, Converso D, Viotti S. Evaluating the psychometric properties of the Maslach Burnout Inventory-Human Services Survey (MBIHSS) among Italian nurses: how many factors must a researcher consider? PLoS One 2014; 9:e114987.

30. Watts J, Robertson N, Winter R, Leeson D. Evaluation of organisational culture and nurse burnout. Nurs Manag (Harrow) 2013; 20:24-9.

31. Frutos Martín M. Relación entre los modelos de gestión de recursos humanos y los niveles de estrés laboral y burnout en los profesionales de enfermería de atención especializada [Tesis de Doctorado]. Léon: Escuela Universitaria de Ciencias de la Salud, Universidad de León; 2014.

32. Cañadas-De La Fuente GA, Vargas C, San Luis C, García I, Cañadas GR, De La Fuente EI. Risk factors and prevalence of burnout syndrome in the nursing profession. Int J Nurs Stud 2015; 52:240-9.

33. Grau A, Suñer R, García MM. Desgaste profesional en el personal sanitario y su relación con los factores personales y ambientales. Gac Sanit 2005; 19:463-70.

34. Vicente-Herrero MT, Torres Alberich JI, Capdevila García L, Gómez JI, Ramírez Îñiguez de la Torre MV, Terradillos García MJ, et al. Trabajo nocturno y salud laboral. Rev Esp Med Legal 2016; 42:142-54.

35. Mansilla Izquierdo F. Manual de riesgos psicosociales en el trabajo: teoría y práctica. 2009 http://www.psicologia-online.com/ebooks/ riesgos/ (accedido el 27/Feb/2018).

36. Fernández-Montalvo J, Piñol E. Horario laboral y salud: consecuencias psicológicas de los turnos de trabajo. Revista de Psicopatología y Psicología Clínica 2000; 5:207-22.

37. Toppinen-Tanner S, Ojajärvi A, Väänänen $A$, Kalimo R, Jäppinen P. Burnout as a predictor of medically certified sick-leave absences and their diagnosed causes. Behav Med 2005; 31:18-27.

38. Li X, Guan L, Chang H, Zhang B. Core selfevaluation and burnout among nurses: the mediating role of coping styles. PLoS One 2014; 9:e115799.

39. Piko BF. Burnout, role conflict, job satisfaction and psychosocial health among Hungarian health care staff: a questionnaire survey. Int J Nurs Stud 2006; 4:311-8.

40. Garrosa E, Moreno-Jiménez B, RodríguezMuñoz A, Rodríguez-Carvajal R. Role stress and personal resources in nursing: a cross-sectional study of burnout and engagement. Int J Nurs Stud 2011; 48:479-89.

41. Bujalance Hoyos J, Villanueva Pardo F, Guerrero Manzano S, Conejo García J, González Valentín A, Sepúlveda Jurado A, et al. Burnout y satisfacción laboral de los profesionales que atienden a pacientes geriátricos. Rev Esp Geriatr Gerontol 2001; 36:32-40.

42. Siegrist J. Adverse health effects of high-effort/ low-reward conditions. J Occup Health Psychol 1996; 1:27-41. 
43. Seidler A, Thinschmidt M, Deckert S, Then F, Hegewald J, Nieuwenhuijsen K, et al. The role of psychosocial working conditions on burnout and its core component emotional exhaustion - a systematic review. J Occup Med Toxicol 2014; 9:10.

44. Jervis LL. Working in and around the 'chain of command': power relations among nursing staff in an urban nursing home. Nurs Inq 2002; 9:12-23.

45. Lee RT, Seo B, Hladkyj S, Lovell BL, Schwartzmann L. Correlates of physician burnout across regions and specialties: a meta-analysis. Hum Resour Health 2013; 11:48

46. Taris TW, Kompier MAJ, Geurts SAE, Houtman ILD, van den Heuvel FFM. Professional efficacy, exhaustion, and work characteristics among police officers: a longitudinal test of the learning-related predictions of the demand: control model. J Occup Organ Psychol 2010; 83:455-74.

47. Lin Y-W. The causes, consequences, and mediating effects of job burnout among hospital employees in Taiwan. J Hosp Adm 2012; 2:1527.

48. Artaza O, Barría MS, Fuenzalida A, Núñez K, Quintana A, Vargas I, et al. Modelo de gestión de establecimientos hospitalarios. Santiago: Ministerio de Salud; 2005
49. Faura i Vendrell T. Síndrome de burn out: enfermería, grupo de alto riesgo. Rev Rol Enferm 1988; XI:53-6.

50. Llorens Gumbau S, Salanova Soria M. Burnout: un problema psicológico y social. Riesgo Labor 2011; 37:26-8.

51. World Health Organization. Guidelines for the primary prevention of mental, neurological and psychosocial disorders. 5. Staff burnout. Geneva: World Health Organization; 1994.

52. World Health Organization. The World Health Report 2000: health systems: improving performance. Geneva: World Health Organization; 2000.

53. Mingote JC, Pérez S. Estrés en la enfermería. El cuidado del cuidador. Madrid: Díaz de Santos; 2003.

54. Gil-Monte PR. El síndrome de quemarse por el trabajo (síndrome de burnout) en profesionales de enfermería. Revista Eletrônica InterAção Psy 2003; 1:19-33.

55. Peiró J. Stress and coping at work: new research trends and their implications for practice. In: Naswall K, Hellgren J, Sverke M, editors. The individual in the changing working life. Cambridge: Cambridge University Press; 2008. p. 284-310. 


\section{Abstract}

The aim of this study was to assess the relationship between psychosocial risks and burnout syndrome in a long-stay hospital in Spain. A cross-sectional study was conducted in 2017, applying the Spanish version of the MBI-HSS and the F-Psico 3.1 questionnaire of Spain's National Institute of Work Safety and Health. The predictive variables were sociodemographic characteristics, modulators, and psychosocial risk factors. The outcome variables were prevalence of burnout and the effects on his subscales. Associations between variables were measured by odds ratio. Burnout was directly associated with psychosocial risks related to workload, psychological demands, participation/supervision, role performance and social support, and consumption of anxiolytics. Meanwhile, protective factors were having children, feeling valued by patients and coworkers, satisfaction at work, optimism, and social support. The associations found on depersonalization were similar but weaker. Low personal fulfillment was directly associated with the psychosocial risks related to length of workweek, limited autonomy and variety/content of work, and role performance and social support. Low personal fulfillment was the subscale with the most modulating and protective sociodemographic variables included marital status, children, night shift, feeling valued by patients and family members, social support, self-efficacy, and optimism. According to our results, there is an association between psychosocial risks and burnout syndrome. Individuals with greater work satisfaction, self-efficacy, and optimism cope better with stress and are less vulnerable to psychosocial risks and burnout.

Profesional Burnout; Mental Fatigue; Occupational Stress; Health Personnel

\section{Resumo}

Nosso objetivo foi avaliar a relação entre os riscos psicossociais e a sindrome de burnout em um hospital espanhol de meia-longa permanência. Foi realizado um estudo transversal em 2017, aplicando a versão espanhola do MBI-HSS e o questionário F-Psico 3.1 do Instituto Nacional de Segurança e Higiene no Trabalho. Variáveis preditoras: características sociodemográficas, moduladoras e fatores de risco psicossocial. Variáveis de resultado: prevalência de burnout e afetação das subfaixas. A associação entre variáveis foi quantificada com odds ratio. A fatiga emocional foi associada positivamente aos riscos psicossociais vinculados a uma carga de trabalho, demandas psicológicas, participação/supervisão, desempenho de funções, relações/apoio social e consumo de ansiolíticos; por outro lado, foram fatores protetores: os filhos, se sentir valorados por pacientes e companheiros de trabalho, satisfação laboral, otimismo e apoio social. As associações encontradas para a despersonalização foram similares, entretanto mais débeis. A baixa realização pessoal foi associada positivamente aos riscos psicossociais vinculados ao tempo trabalhado, autonomia, variedade/conteúdo do trabalho, desempenho de funções e apoio social; foi a subfaixa que mostrou maior número de variáveis sociodemográficas/moduladoras protetoras: estado civil, ter filhos, trabalhar de noite, sentirse valorizado por pacientes e membros da família, ilusão pelo trabalho, apoio social, auto-eficácia e otimismo. Segundo nossos resultados, existe uma associação entre os riscos psicossociais e a sindrome de burnout. Os indivíduos com maior satisfação laboral, auto-eficácia e otimismo afrontam melhor o stress e são menos vulneráveis aos riscos psicossociais e ao burnout.

Esgotamento Profissional; Fadiga Mental; Estresse Ocupacional; Pessoal de Saúde
Recibido el 01/Nov/2017

Versión final presentada el 08/Jun/2018

Aprobado el 19/Jul/2018 